\title{
Expansion Velocities and Kinematic Ages of Compact Symmetric Objects
}

\author{
Antonis Polatidis ${ }^{* \dagger}$ \\ Joint Institute for VLBI in Europe, Postbus 2, 7990AA Dwingeloo, The Netherlands \\ E-mail: polatidis@jive.nl
}

For the past decade, multi-epoch VLBI observations of Compact Symmetric Objects (CSOs) with temporal coverage of a few years to a couple of decades have enabled the measurement of the increase of hotspot separation which implied an expansion of the lobes. Given the long temporal coverage it is probably safe to assume that they represent the average lobe expansion velocity and hence the mean growth rate of the sources.

Since the observed symmetry hints that there are no major projection effects, first order estimates of the "kinematic" ages of the order of a few decades to a few thousand years have been calculated for more than 25 sources so far. Long term monitoring, with three or more observations separated by at least a few years to even a decade or more, are necessary to obtain an accurate or indeed just one kinematic measurement.

Whereas in most sources, radial expansion (ie motion along the hotspot-to-hotspot direction) is observed, there are cases with more pronounced side-to-side velocities (e.g. 1031+567 [9], $0108+388$ [6]. Recently [7] reported apparent 'contraction' in J11584+2450, a similar effect to that reported for the CSO 0646+600 [1].

Here I discuss multi-epoch VLBI observations of CSOs from the " $200 \mathrm{mJy}$ sample", that illustrate examples of straightforward measurement of lobe expansion, the difficulty to obtain quantitative age measurements in the absence of compact hotspots and a source where we observe recurrent radio activity.

The 9th European VLBI Network Symposium on The role of VLBI in the Golden Age for Radio Astronomy and EVN Users Meeting

September 23-26, 2008

Bologna, Italy

\footnotetext{
*Speaker.

${ }^{\dagger}$ This work is done in collaboration with M. Marchã, M. Bondi and S. Anton
} 


\section{Kinematic observations of CSOs in the $200 \mathrm{mJy}$ sample}

Proving the fragility of simple (ie two frequency) spectral classification, the "200 mJy sample" [4], which consists of low redshift, flat spectrum radio sources selected from JVAS and mainly identified with BL Lac objects and radio galaxies, yielded 9 sources with CSO morphology (Bondi et al. 2004 and Polatidis in prep) of which three were already known: 0035+227, 4C31.04, 0729+562, 0902+468, NGC3894, 1245+676, OQ208, 1558+595 and 1755+626.

Subsequent monitoring to detect any motion has up to now covered three epochs over 6 years and has yielded expansion velocities, and a few negative results. I discuss below examples of these measurements, made from observations at $5 \mathrm{GHz}, 8 \mathrm{GHz}$ and $15 \mathrm{GHz}$ with the VLBA and global VLBI arrays on 1998.56, 2000.59, 2001.53 and 2004.99

\section{$1.10035+227$}

The $\mathrm{z}=0.096$ radio galaxy $0035+227$ [4] shows a 22 parsec large, mainly triple structure with a compact flat spectrum $(\alpha=-0.23)$ middle component situated between two steeper spectrum resolved "mini-lobes" with embedded hotspots $\left(\alpha_{N W}^{\text {hotspot }}=1.25\right.$ and $\left.\alpha_{S E}^{\text {hotspot }}=0.8\right)$. The core is connected to the lobes with low level emission, suggesting symmetric jets. Using three epoch observations at $5 \mathrm{GHz}$ we measure an increase in the angular distance between the hotspots of 0.041 mas/yr, implying an expansion speed of $0.15 h^{-1} \mathrm{c}$ and yielding a kinematic age of $\sim 450$ years.

\subsection{The double-double $1245+676$}

$1245+676$ is giant double-lobed radio galaxy with an angular size of $12.4 \mathrm{arcmin}\left(\sim 970 \mathrm{~h}^{-1}\right.$ kpc, eg. [3]). Its luminosity and arcsec-scale morphology classify it as an FRII galaxy with the exception that the arcsec-scale core dominates the total flux density and has a GPS spectrum. There are no compact hotspots in its arcsec-scale lobes.

At parsec scale resolutions this central component is resolved in two steep spectrum 'minilobes $\left(\alpha_{\text {north }}=-1.03, \alpha_{\text {south }}=-1.2\right)$ with a total size of $9.6 h^{-1} \mathrm{pc}$. There is no indication of a compact, inverted spectrum core at high frequencies $(22 \mathrm{GHz}, 43 \mathrm{GHz},[5])$. Combining our observations with unpublished observations from 1989-1990 we find that the hotspots embedded in the two lobes are separating with a rate of $0.16 h^{-1}$ c yielding a kinematic age of $\sim 188$ years for the inner structure.

The fact that we see no emission between the inner parsec scale CSO and the outer lobes, either in the form of low level emission or a kpc-scale jet and the lack of hotspots in the outer lobes, implies that feeding of the lobes with electrons stopped at a significant time ago (probably more than $10^{4}$ years) and that the parsec scale emission is a new stage of radio activity.

\section{$1.31558+595$}

This $\mathrm{z}=0.06$ galaxy shows a $\sim 10 \mathrm{pc}$ large, two-sided emission extending consistent with twin jets. A prominent jet emerges to the northwest of a compact (at $15 \mathrm{GHz})$ flat spectrum $(\alpha=0.04)$ core (A) and q weak counterjet, heavily resolved at $15 \mathrm{GHz}$, appears to the south, south-west of the core. The jets contain components with spectral indices ranging from $\alpha=0.44$ to $\alpha=0.78$. In our 3-epoch 5GHz VLBI observations (1998-2004), a component in the jet moves with a velocity of $5 h^{-1}$ c. while a component in the counterjet moves with $2.21 h^{-1}$ c. Since there is no evidence 

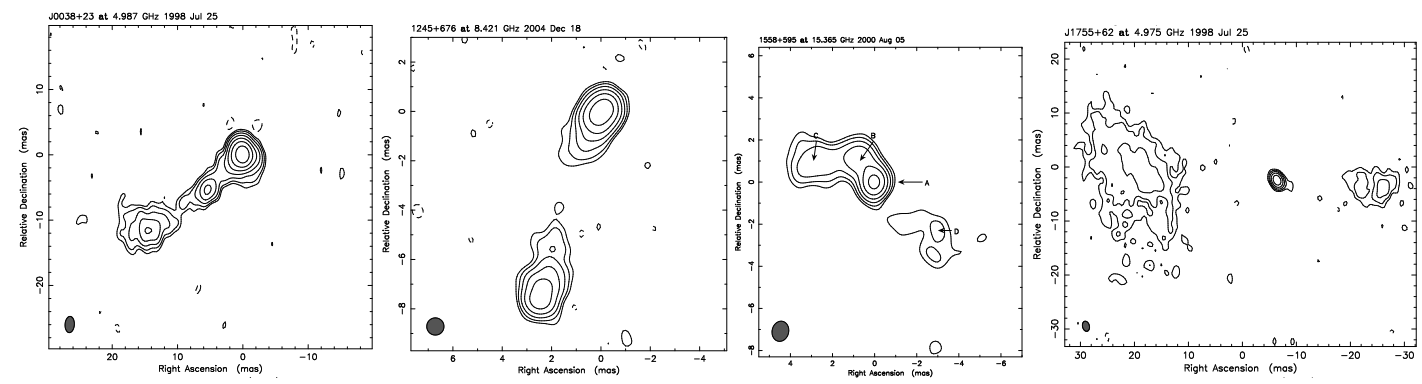

Figure 1: Images of $0035+227$ at $5 \mathrm{GHz}$ (epoch 1998.56), 1245+676 at $8 \mathrm{GHz}$ (epoch 2004.9), 1558+595 at $15 \mathrm{GHz}$ (epoch 2000.59 ) and $1755+626$ at $5 \mathrm{GHz}$ (epoch 1998.56)

for hotspots, these velocities are not representative of the source age and the source is analogous to $1946+708$ [8] where bi-directional motion in symmetric jets has been detected.

\section{$1.41755+626$ (NGC6521)}

This elliptical galaxy $(\mathrm{z}=0.024)$, shows only a 20 parsec large structure with two extended and very steep spectrum (resolved out at $15 \mathrm{GHz}$ ) lobes straddling a weak, inverted spectrum core. There is no sign of a compact component that could be interpreted as a hotspot in either lobe, hence it is very difficult to obtain quantitative measure of the lobe kinematics either via model-fitting or direct image comparison. We estimate an upper limit of $0.03 h^{-1} \mathrm{c}$ of the lobe expansion velocity.

\section{References}

[1] Akujor, C.E., Porcas, R.W., Smoker, J.V., Apparent contraction in the mas radio structure of the 'red quasar' Q0646+600, A\&A, 306, 391, 1996

[2] Bondi, M., Marchã, M. J., Polatidis, A., Dallacasa, D., Stanghellini, C., Antón, S. VLBA polarization observations of BL Lac objects and passive elliptical galaxies, MNRAS, 352, 112. 2004

[3] Lara, L., Cotton, W. D., Feretti, L., Giovannini, G., Marcaide, J. M., Márquez, I., Venturi, T. A new sample of large angular size radio galaxies. I. The radio data, A\&A, 370, 409, 2001.

[4] Marcha, M. J. M., Browne, I. W. A., Impey, C. D., \& Smith, P. S. Optical spectroscopy and polarization of a new sample of optically bright flat radio spectrum sources, MNRAS, 281, 425, 1996.

[5] Marecki, A., Barthel, P. D., Polatidis, A., \& Owsianik, I. 1245+676 - a CSO/GPS source being an extreme case of a double-double structure, PASA, 20, 16, 2003

[6] Owsianik, I., Conway, J. E., \& Polatidis, A. G. Evidence for recurrent activity of age 370 years in the compact symetric radio source 0108+377 A\&A, 336, L37, 1998

[7] Tremblay, S. E., Taylor, G. B., Helmboldt, J. F., Fassnacht, C. D., \& Pearson, T. J. A Shrinking Compact Symmetric Object: J11584+2450?, ApJ, 684, 153, 2008

[8] Taylor, G. B., \& Vermeulen, R. C. Bi-Directional Relativistic Jets of the Radio Galaxy 1946+708: Constraints on the Hubble Constant, ApJl, 485, L9, 1987

[9] Taylor, G. B., Marr, J. M., Pearson, T. J., \& Readhead, A. C. S.Kinematic Age Estimates for Four Compact Symmetric Objects from the Pearson-Readhead Survey, ApJ, 541, 112, 2000 